Proceedings of the 2003 IEEE/RSJ

Intl. Conference on Intelligent Robots and Systems

Las Vegas, Nevada · October 2003

\title{
An Efficient Strategy for Rapidly Finding an Object in a Polygonal World
}

\author{
Alejandro Sarmiento Rafael Murrieta Seth A. Hutchinson \\ Beckman Institute for Advanced Science and Technology \\ University of Illinois at Urbana-Champaign \\ \{ asarmien, murrieta, seth \} @ uiuc.edu \\ Urbana, Illinois 61801 \\ USA
}

\begin{abstract}
In this paper we propose an approach to solve the problem of finding an object in a polygon which may contain holes. We define an optimal solution as the route that minimizes the expected time it takes to find said object.

The object search problem is shown to be NP-hard by reduction, therefore, we propose the heuristic of an utility function, defined as the ratio of a gain over a cost and an greedy algorithm in a reduced search space that is able to explore several steps ahead without incurring in too high a computational cost.

This approach was implemented and simulation results are shown.
\end{abstract}

\section{INTRODUCTION}

The problem of determining a good strategy to accomplish a visibility-based task such as environment modeling [2], pursuit-evasion [5] [6], or object finding [4] [13], is a very challenging an interesting research area. Specially when the sensors are not static but rather are carried by mobile robots.

We are interested in the problem of finding an object. Our goal is to find an efficient strategy to perform the object search. The possible applications have a wide range, from finding a specific piece of art in a museum to search and rescue injured people inside a building.

In general, the robot will not be able to see the whole environment in a single sensing. Therefore, more than one perceptions will be needed to completely cover the environment. There are several schemes to generate and combine these perceptions.

One approach is to have the robot continuously sense the world as it moves along a given trajectory, thereby scanning the environment in a continuous fashion. In this case, it is not clear how to generate a globally optimal trajectory for a given criterion. A greedy strategy could use the gradient of the new visibility information to guide the search. This strategy could be based on critical events such as crossing lines in an aspect graph based on perspective projection [13].

Another approach is to make the robot sense the environment only at specific locations. This changes the nature of the problem from continuous to discrete, with information arriving in blocks. This also introduces the problem of generating an "appropriate" set of sensing locations.

There are several criteria for determining the goodness of this set. For example, the minimal number of locations (art gallery problem [8]), locations along the shortest path that covers the whole environment (shortest watchman path [12]), and so on.

In this paper we will assume that the set of locations is given as input - they will not be generated automatically. In any case, once the sensing locations are known, it is still necessary to visit those locations in a specific order to minimize the expected time to find the object. This transforms the object search into a combinatorial problem.

In this paper our objective is to generate an exploration strategy based on the given sensing locations that finds the object as quickly as possible. That is, a strategy that minimizes the expected time it takes to find it (as explained in the next section). Under this definition of optimality, we have proven that the best exploration strategy is not necessarily the one that mininizes the distance traveled by the robot [11].

\section{PROBLEM DEFINITION}

In general terms, we define the problem of searching for an object as follows: Given a mobile robot with some kind of sensing capabilities, a completely known environment and an object sitting somewhere in the world, develop a motion strategy for the robot to find the object in the least amount of time.

At this point we are not concerned with the geometry of the robot or the capabilities of the sensor (field of view, range, resolution and so on). For now, we consider only a point robot with an omnidirectional, infinite range sensor. These assumptions are made to simplify the analysis and to better understand the problem. In the future, we will address the more complex cases.

We established that the environment $W$ is known and modeled as a polygon which may contain holes. All these obstacles generate both motion and visibility constraints.

Furthermore, we assume that the probability of the object being in any specific point is evenly distributed 
throughout the polygon's interior. Therefore, the probability of the object being in any subset $R \subseteq W$ is proportional to the area of $R$.

We also assume that we are given a set of locations $L$ (also known as guards from the art gallery problem [12]) from which every point in $W$ can be seen. The visibility region of location $L_{j}$, denoted $V\left(L_{j}, W\right)$, is the set of points in $W$ that have a clear line of sight to $L_{j}$ (the line segment connecting them does not intersect the exterior of $W$ ). The set $L$ is chosen so that the associated visibility regions define a cover of $W$. This means that their union adds up to the whole environment $W$.

$$
\bigcup_{j} V\left(L_{j}, W\right)=W
$$

We do not require nor assume the set $L$ to be minimal.

Our exploration protocol is as follows: the robot always starts at a particular location in $L$ (the starting point) and visits the other locations as time progresses (it follows the shortest paths between them). It only gathers information about the environment (sensing) when it reaches one of these locations - it does not sense while moving. We describe the route followed by the robot as a series of locations $L_{i_{k}}$ that starts with the robot's initial location and includes the other locations once. It is important to note that while $L_{j}$ refers to locations in the environment, $L_{i_{k}}$ refers to the order in which those locations are visited. That is, the robot always starts at $L_{i_{0}}$, and the $k$-th location it visits is referred to as $L_{i_{k}}$. Obviously, every $L_{i_{k}}$ has a corresponding $L_{j}$ in the environment, but their indices need not match.

For any route $S$, we define the time to find the object $T$ as the time it takes to go through the locations - in order - until the object is first seen. We assume that the robot will be able to identify the object from any given viewpoint and that there are no other objects that could be mistaken as the searched object [3].

Our goal is to find the route that minimizes the expected value of the time it takes to find the object

$$
E[T \mid S]=\sum_{j} t_{j} P\left(T=t_{j}\right)
$$

where

$$
P\left(T=t_{j}\right)=\frac{\operatorname{Area}\left(V\left(L_{i_{j}}, W\right) \backslash \bigcup_{k<j} V\left(L_{i_{k}}, W\right)\right)}{\operatorname{Area}(W)}
$$

Where $t_{j}$ is the time it takes the robot to go from its initial position - through all locations along the route - until it reaches the $j$-th visited location $L_{i_{j}}$, and $P\left(T=t_{j}\right)$ is the probability of finding the object there. Since the robot only senses at specific locations, we also denote this probability of finding the object at location $L_{i_{j}}$ as $P\left(L_{i_{j}}\right)$.
Explicitly, the probability of finding the object from a given location is proportional to the visibility polygon of that region $\left(V\left(L_{i_{j}}, W\right)\right)$ minus the already explored space up to that point $\left(\bigcup_{k<j} V\left(L_{i_{k}}, W\right)\right)$.

\section{PROPOSED SOLUTION}

Since we assume that we are given a set of sensing locations that completely cover the environment, we are interested in finding an order of visiting those locations - the problem becomes a combinatorial search. In this section we present two algorithms for such a task. The first one is a traditional graph search that finds the optimal ordering but is intractable. The second is a greedy algorithm that can be computed in polynomial time and yields good results.

In general, the robot will not be able to travel between two locations by following a straight line. In this cases, we use a reduced visibility graph [9] and Dijkstra's Algorithm to follow the shortest path between them.

\section{A. Algorithm for Optimal Ordering}

Given a set of locations $L$ that are guards to a polygonal region $W$, there exists an algorithm for computing the route that minimizes the expected time to find the object. It is described hereafter.

Construct a complete weighted graph as follows:

(1) For each location $L_{j}$, create a node $N_{j}$ in the graph.

(2) For each pair of nodes $N_{j}$ and $N_{k}$, add an edge with variable weight $W_{j k}$.

(3) The weight $W_{j k}$ is dynamic, meaning it depends on the route followed by the robot before reaching $N_{j}$. These weights are calculated on-line.

The weight $W_{j k}$ should correspond to the increase in expected time $\Delta E[T]$ the robot incurs by going from $L_{j}$ to $L_{k}$. This is a function of the time in which it arrives at $L_{k}$, which in turn depends on the route followed by the robot up to that point.

In this graph, we need to find the path of minimum cost that starts at the robot's initial location $L_{i_{0}}$ and includes all other locations. This can be accomplished with a Branch and Bound graph search. This search strategy maintains a list of nodes to be opened ordered by their accumulated cost. The next node to be expanded is always the head of the list, the one whose accumulated cost is currently minimal.

When a node is expanded, only those nodes that are adjacent and not already included in the current path are considered children. The added cost $W_{j k}$ of expanding a child $N_{k}$ from its parent $N_{j}$ is

$$
\begin{aligned}
W_{j k} & =\operatorname{Time}\left(N_{k}\right) \cdot P\left(L_{k}\right) \\
\operatorname{Time}\left(N_{k}\right) & =\operatorname{Time}\left(N_{j}\right)+\operatorname{Speed} \cdot \operatorname{Dist}\left(L_{j}, L_{k}\right)
\end{aligned}
$$

Then, the accumulated cost for the child is

$$
\operatorname{Cost}\left(N_{k}\right)=\operatorname{Cost}\left(N_{j}\right)+W_{j k}
$$


Initially, the Branch and Bound list contains only the starting robot location. Then, the head of the list is expanded and its children added to the ordered list until a solution is found - a path that contains all locations in $L$. When this happens, the currently best nodes continue to be expanded until

(a) A lower cost solution is found, in which case the better solution is saved and the process continues, or

(b) The lowest cost node is worse than the current solution. In this case we know that this solution is optimal.

This algorithm finds the optimal solution - the one that minimizes the expected time to find the object. Unfortunately, its space and time complexities are not of polynomial order. Furthermore, the problem itself is intractable, more specifically, NP-hard.

\section{B. Reduction from an NP-hard problem}

The Minimum Weight Hamiltonian Path Problem, known to be NP-hard [1], can be reduced to the problem of finding the optimal visiting order of sensing locations which minimizes the expected time to find an object.

In order to make a formal reduction, we abstract the concept of environment and visibility regions. We only consider a set of locations which have an associated probability of finding the object and are independent of each other.

The reduction consists in defining the distance between the sensing locations as the edge weights of the Minimum Weight Hamiltonian Path Problem and setting the probabilities uniformly (same value for all).

Since the probabilities are set uniformly, the route that minimizes the expected time will be the exactly the same as the one that minimizes the distance traveled. This happens because the expected value of the time to find an object is determined only by the time it takes to reach locations along the route. Since time is proportional to distance, the route that minimizes time will also minimize the distance.

Given that the solutions to both problems are the same ordering of locations, finding a polynomial algorithm to solve these instances of the defined problem would also solve the Minimum Weight Hamiltonian Path Problem in polynomial time. Thereby proving that the proposed problem is NP-hard.

\section{Utility Heuristic}

Since trying to find an optimal solution is a futile effort, we decided to implement an iterative greedy strategy. One that tries to achieve a good result in one (or just a few) steps at a time.

In the obvious version of this algorithm the next location to visit is chosen as the one that causes the least increase in the partial calculation of (1) along the current route. That is, at each step of the route, calculate how much would the expected value of the time to find the object increase for going to the remaining locations and then choose the least increase. This has $O\left(n^{2}\right)$ complexity, because each step has to consider every available location.

This algorithm performs poorly. We believe this happens because the product in (1) makes locations with low probability be preferred and visited first, which seems contrary to what should be done.

For this reason, we propose an alternate greedy algorithm, called utility greedy, that tries to maximize an utility function. This function measures how convenient it is to visit a determined location from another, and is defined as follows:

$$
U\left(L_{j}, L_{k}\right)=\frac{P\left(L_{k}\right)}{\operatorname{Time}\left(L_{j}, L_{k}\right)}
$$

This means that if the robot is currently in $L_{j}$, the utility of going to location $L_{k}$ is proportional to the probability of finding the object there and inversely proportional to the time it must invest in traveling.

A robot using this function to determine its next destination will tend to prefer locations that are close and/or locations where the probability of finding the object is high. Intuitively, it is convenient to follow such an strategy, but its relationship with the expected value minimization will be more evident after the following analysis.

Consider a definition of expectation for a non-negative random variable, such as time, from [10]

$$
E[T]=\int_{0}^{\infty} P(T>t) d t
$$

This is equivalent to

$$
E[T]=\int_{0}^{\infty}(1-P(T \leq t)) d t=\int_{0}^{\infty}\left(1-F_{T}\right) d t
$$

Where $F_{T}$ is a cumulative distribution function.

In our problem, every valid trajectory defines a particular cumulative distribution function of finding the object, $F_{T}$. Since we are dealing with a discrete problem, the distributions are only piecewise continuous with the discontinuities being the times at which the robot reaches the distinct locations along the route, as shown in Fig. 1a.

By (3), we know that the expected value of a random variable with distribution $F_{T}$ is the area under the curve $1-F_{T}$, shown in Fig. 1b. This area is the value we want to minimize.

One method for making this area small is to have the time intervals as small as possible and the probability changes (down step) as large as possible. This is the notion that our utility function in (2) captures; its value is larger when the probability of finding the object in a particular location is high (large down step) and/or when the location is near (small time interval). 


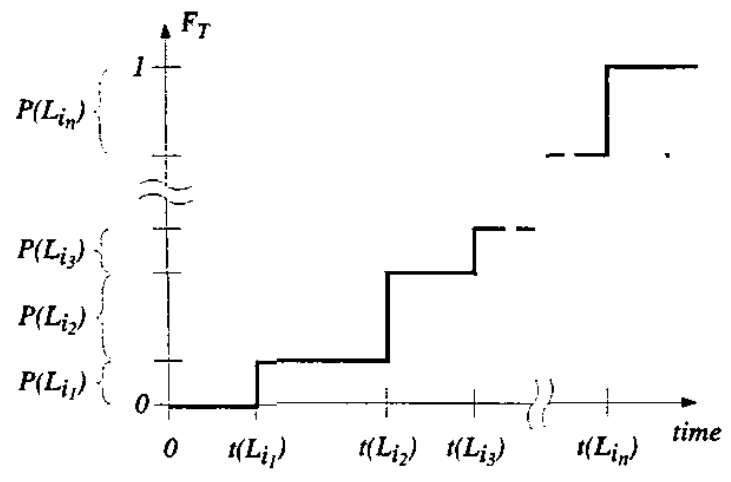

(a)

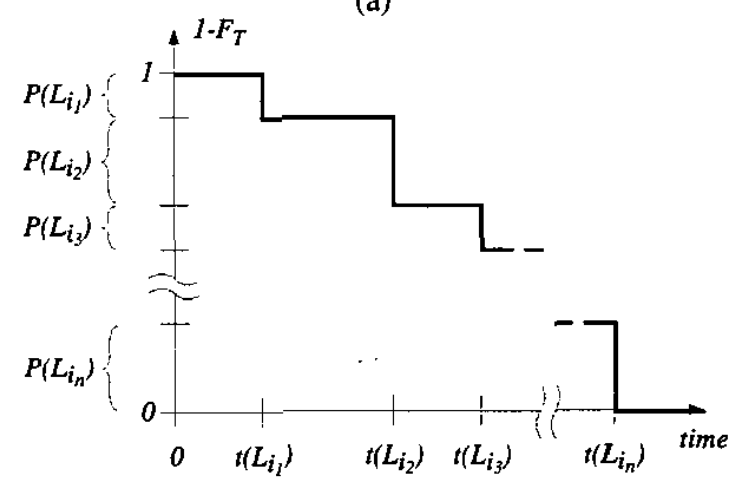

(b)

Fig. 1. Defi ned cumulative distribution functions. (a) $F_{T}$ (b) $1-F_{T}$

\section{Efficient Utility Greedy Algorithm}

The utility function in (2) is sufficient to define a 1step greedy algorithm. At each step, simply evaluate the utility function for all available locations and choose the one with the highest value. This algorithm has a running time of $O\left(n^{2}\right)$.

However, it might be convenient to explore several steps ahead instead of just one to try to "escape local minima" and improve the quality of the solution found. The downside of this idea is that it usually increases the complexity of the algorithm by a factor of $O(n)$ for each step ahead.

To reduce this effect we propose a second heuristic that reduces the branching factor. The heuristic is that the children of each location can only be those other locations that are not strictly dominated according to the two variables in the utility function. As seen from the $j$-th location $L_{j}$, a location $L_{k}$ strictly dominates another $L_{l}$ if both of the following conditions are true

$$
\begin{aligned}
P\left(L_{k}\right) & >P\left(L_{l}\right) \\
\operatorname{Dist}\left(L_{j}, L_{k}\right) & <\operatorname{Dist}\left(L_{j}, L_{l}\right)
\end{aligned}
$$

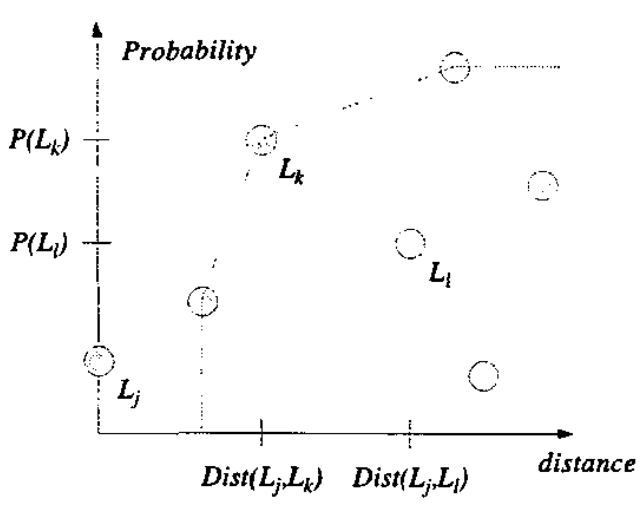

Fig. 2. Location dominance

Graphically, this is shown in Fig. 2. It is straightforward that dominating locations will lie on the convex hull of the remaining set of locations when plotted on the probability vs. distance plane. The endpoints of this partial convex hull are not considered as candidates since they are not defined locations.

By only considering a subset of the remaining locations at each step, we are reducing the branching factor, making it possible to explore more steps ahead without incurring in too high a computational cost. Of course, there is no guarantee that the optimal solution is indeed a member of this reduced search space or even that this will yield better results. However, we have found it to be a good heuristic in practice, as described in the next section.

The full algorithm consists in iteratively exploring several steps ahead, choosing the most promising route up to that point and starting over from there. For $n$ locations, if the branching factor is $B$, a tree of height $\log _{B}(n)$ can be explored in linear time. This creates a partial route of length $\log _{B}(n)$. Since a solution should be of length $n$, the process needs to be repeated $\frac{n}{\log _{B}(n)}$ times for the complete route. This is depicted in Fig. 3 . The big triangle represents the tree that would be generated if a complete exploration was made, whereas the small triangles represent the trees that are actually generated (explored) by the algorithm.

Thus, our final algorithm is as follows:

(1) For the last location along the current solution (initially just the robot starting location) explore the possible routes (create a tree breadth-first) until the number of nodes is of order $O(n)$.

(2) For each node that needs to be expanded, compute the set of locations that are not strictly dominated by others and only choose those as children. This can be done with a convex hull algorithm in $O(n \log (n))$.

(3) When the number of nodes in the exploration tree has reached order $O(n)$, choose the best leaf accord- 


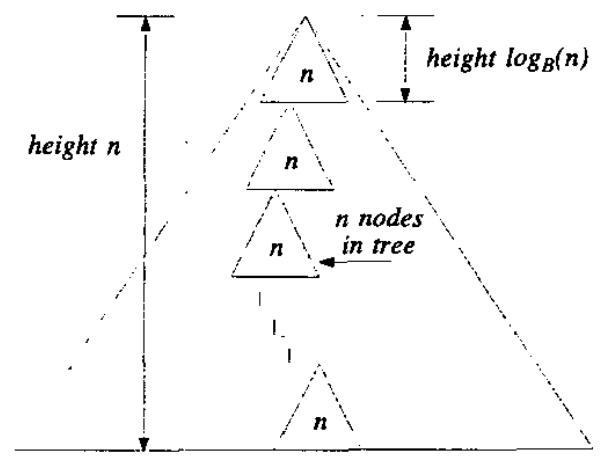

Fig. 3. Exploration algorithm

ing to the heuristic in (2), discard the current tree and start over with the best node as root.

This has to be repeated several times to generate a complete route, therefore the total complexity of the algorithm is

$$
O\left(n \cdot n \log (n) \cdot \frac{n}{\log (n)}\right)=O\left(n^{3}\right)
$$

Of course, this result depends on the number of dominating locations being significantly smaller than $n$ on average. Which may be difficult to determine for a specific problem. We know, for example, that the expected number of points on the convex hull of a set sampled uniformly from a convex polygon is of order $O(k \log (n))$ for a $k$ sided polygon [7]. In the worst case, when the branching factor is not reduced at all, our algorithm only explores one step at a time and has a running time of

$$
O(n \cdot n \log (n) \cdot n)=O\left(n^{3} \log (n)\right)
$$

This analysis only considers the time complexity of the search algorithm itself. It does not include the time complexity of performing polygon clipping operations. These are needed every step of the algorithm because they are used to calculate the probability of finding the object at any given location (which depends on the route followed up to that point).

\section{-IV. SIMULATION RESULTS}

For our simulations, we implemented routines for computing visibility polygons, the reduced visibility graph and shortest paths (Dijkstra's Algorithm). For calculating the union of visibility regions, we used the $g p c$ library developed by Alan Murta [14].

This section presents the simulation results for the polygonal world shown in Fig. 4. The black regions correspond to the obstacles, the small circles to the sensing locations (guards) given as input and the grey region is the visibility polygon of the starting location.

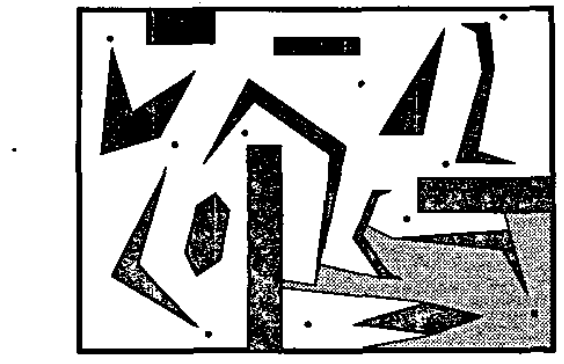

Fig, 4. Test polygonal world

For this instance, we generated the sensing locations manually. While we tried to find as few as possible, they do not correspond to any kind of optimal criteria.

For the following routes, we show the expected value of the time it takes to find the object following that particular route, and its total length. These values are given in arbitrary units, what really. matters in the relative value differences between the routes. The execution times are in seconds for a regular PC workstation.

For this polygonal world, we computed three routes. The first one is the route that minimizes the expected value of the time to find the object (the optimal solution). For purposes of comparison, we also computed the route that minimizes the distance traveled, and finally, we show the route generated by our heuristic algorithm.

We were able to solve thie optimal cases because the number of sensing locations is relatively small (10).

Fig. 5 shows the route that minimizes the expected value of the time to find the object - the optimal solution to our problem. For this route the expected value is 943.21 with a total distance traveled of 2783.20 . This result took 892.82 seconds to compute.

Fig. 6 shows the route that minimizes the distance traveled from the starting location. In this case, the expected value of the time to find the object is 994.79 with a total distance of 2273.09. This route was computed in 488.87 seconds. This result further shows that the best strategy to find an object as quickly as possible on average, is not

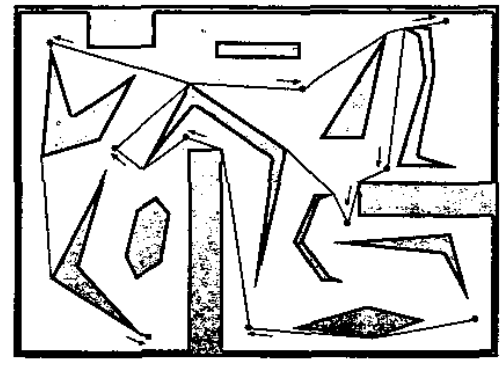

Fig. 5. Route that minimizes the expected time to fi nd the object 


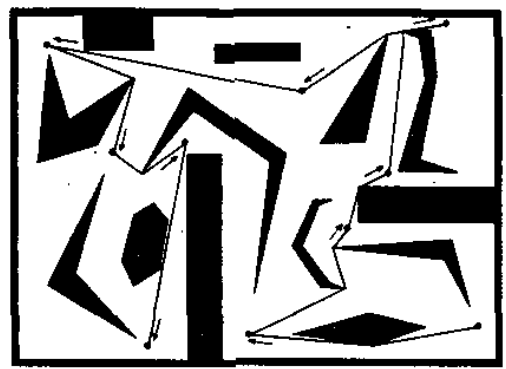

Fig. 6. Route that minimizes the distance traveled

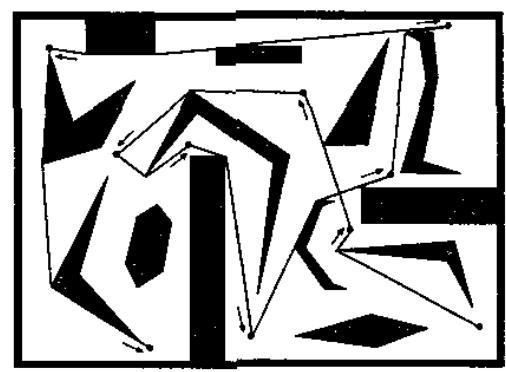

Fig. 7. Route generated by the utility heuristic algorithm

the one that minimizes the distance traveled.

Fig. 7 shows the route generated by our heuristic algorithm. The expected value along this route is 982.21 with a total distance traveled of 2970.43 . This result was obtained in only 0.44 seconds.

For this particular example, the expected value of the time to find the object along the route obtained by our heuristic algorithm is slightly smaller (by $1.2 \%$ ) than along the route that minimizes the distance traveled. Of course, the length of the route is larger (by about $30 \%$ ).

With respect to the optimal solution, the route generated by our algorithm is worse in both expected value of the time to find the object (by $4.1 \%$ ) and distance travelled (by $6.7 \%$ ). However, in execution time, our algorithm is more than 2000 times faster.

\section{CONCLUSIONS}

In this paper we proposed an efficient approach to solve the problem of searching an object in a polygonal environment. We defined an optimal solution as the route that minimizes the expected time it takes to find the object.

The problem itself was shown to be NP-hard by reduction, therefore, we proposed the heuristic of an utility function, defined as the ratio of a gain (increase in a cumulative distribution function) over a cost (travel time).

We also proposed an greedy algorithm in a reduced search space that is able to explore several steps ahead without incurring in too high a computational cost.
We showed experiments in simulation that suggest that the quality of the routes generated by our algorithm is close to the optimal solutions.

Future work will consist in the development of an approach to generate a set of sensing locations automatically. The set should be "helpful" to the problem of minimizing the expected time to find an object. It seems that desirable properties include low cardinality and low dispersion. A formal definition of these properties is a major part of the solution to the problem.

\section{REFERENCES}

[1] Garey, M.R. and D.S. Johnson, Computers and Intractability, W. H. Freeman and Company, 1979.

[2] González-Baños, H.H. and J.C. Latombe, "Navigation Strategies for Exploring Indoor Environments," to appear in Int. Journal of Robotics Research.

[3] Lacroix, S., P. Grandjean and M. Ghallab, "Perception Planning for a Multi-Sensory Interpretation Machine," in Proc. IEEE Int. Conf. on Robotics and Automation 1992.

[4] LaValle, S.M. et al, "Finding an Unpredictable Target in a Workspace with Obstacles," in Proc. IEEE Int. Conf. on Robotics and Automation 1997.

[5] LaValle S.M. et al, "Motion Strategies for Maintaining Visibility of a Moving Target," in Proc. IEEE Int. Conf. on Robotics and Automation 1997.

[6] Murrieta-Cid, R., H.H. González-Baños and B. Tovar, "A Reactive Motion Planner to Maintain Visibility of Unpredictable Targets," in Proc. IEEE Int. Conf. on Robotics and Automation 2002.

[7] Preparata, F.P. and M.I. Shamos, Computational Geometry: an Introduction, Springer-Verlag New York, 1985.

[8] O'Rourke, J., Art Gallery Theorems and Algorithms, Oxford University Press, 1987.

[9] Rohnert, H., "Shortest Paths in the Plane with Convex Polygonal Obstacles," Information Processing Letters, 23:71-76, 1986.

[10] Ross, S.M., Introduction to Probability and Statistics for Engineers and Scientists, Wiley, 1987.

[11] Sarmiento, A., R. Murrieta and S.A. Hutchinson, "A Strategy for Searching an Object with a Mobile Robot," accepted in Int. Conf. on Advanced Robotics 2003.

[12] Shermer, T.C., "Recent Results in Art Galleries," Proc. of the IEEE, Vol. 80, issue 9, September 1992.

[13] Tovar, B., S.M. LaValle and R. Murrieta-Cid, "Optimal Navigation and Object Finding without Geometric Maps or Localization," accepted in IEEE Int. Conf. on Robotics and Automation 2003.

[14] Vatti, B.R., "A Generic Solution to Polygon Clipping," Communications of the ACM, 35(7), pp.56-63, July 1992. 